\title{
Evolvability Suppression to Stabilize Far-Sighted Adaptations
}

\author{
Lee Altenberg \\ Information and Computer Sciences, University of Hawai'i at Manoa, \\ Honolulu, HI U.S.A. \\ (808) 875-0745, Internet: altenber@hawaii .edu
}

Keywords: evolvability, metapopulation, hierarchies, cheats, far-sighted ${ }^{1}$

\begin{abstract}
The opportunistic character of adaptation through natural selection can lead to 'evolutionary pathologies' - situations in which traits evolve that promote the extinction of the population. Such pathologies include imprudent predation and other forms of habitat over-exploitation or the 'tragedy of the commons', adaptation to temporally unreliable resources, cheating and other antisocial behavior, infectious pathogen carrier states, parthenogenesis, and cancer, an intra-organismal evolutionary pathology. It is known that hierarchical population dynamics can protect a population from invasion by pathological genes. Can it also alter the genotype so as to prevent the generation of such genes in the first place, i.e. suppress the evolvability of evolutionary pathologies? A model is constructed in which one locus controls the expression of the pathological trait, and a series of modifier loci exist which can prevent the expression of this trait. It is found that multiple 'evolvability checkpoint' genes can evolve to prevent the generation of variants that cause evolutionary pathologies. The consequences of this finding are discussed.
\end{abstract}

\section{Introduction}

Adaptation through natural selection is an opportunistic process, in that it is driven by the selective forces of the immediate moment, upon the individual organism. Yet traits that provide immediate advantage to the individual may be detrimental to the population or species, or detrimental over longer time scales. This has been understood since Darwin. Conversely, traits may impose an immediate disadvantage to the individual, yet be advantageous to the population or species, or advantageous over longer time scales. Whether or how such 'far-sighted traits' can evolve has been a challenge for evolutionary mechanics.

\footnotetext{
${ }^{1}$ Copyright (C)2005 by Lee Altenberg. Published in Artificial Life 11 (4): 427-443, special issue on Dynamical Hierarchies.
} 
'Far-sighted' traits includes such phenomena as altruistic behavior-behavior that benefits not its carrier, but other individuals. Other phenomena include cooperation, social organization, prudent predation, population regulation, and multicellular organization.

The converse of 'far-sighted' traits could be called 'short-sighted', 'cheats', (Maynard Smith, 1964), 'selfish', or 'greedy'. Interestingly, in computer science, the term 'greedy' is used to describe algorithms that are 'short-sighted' in that they respond only to immediate conditions without regard to long-term consequences. It is well known that for certain problems, greedy algorithms can lead to outcomes that are ultimately suboptimal.

Examples of short-sighted adaptations include:

- cheating, defection, and other antisocial behavior,

- meiotic drive (Lewontin, 1962),

- parthenogenesis (Griffiths and Butlin, 1995),

- overpopulation (Wynne-Edwards, 1962),

- imprudent predation (Rosenzweig, 1972) and other forms of habitat over-exploitationthe 'tragedy of the commons' (Hardin, 1968),

- cannibalism (Hamilton, 1970),

- cancer (the organism being the population) (Nunney, 1999a; Stoler et al., 1999),

- adaptation to temporally unreliable resources (Kauffman and Johnsen, 1991),

- viable but infectious pathogen carrier states (Kirchner and Roy, 1999),

- evolution of endosymbionts to the detriment of host (Wallace, 1999).

An interesting example of how a straightforward trait such as longevity can become a short-sighted trait is explored by Kirchner and Roy (1999) in the situation of chronic infection. They begin with the observation that longer life spans can result in larger reservoirs of persistently infected hosts, and these reservoirs can in turn reduce population size. While longer life spans provide a reproductive advantage to the individual, in this situation they impose a viability disadvantage to the population. Thus, under certain ecological circumstances, even longevity can be a short-sighted trait.

\subsection{Dynamical Hierarchies}

'Far-sighted' traits by their very definition pose mechanistic difficulties for their evolution, since they may impose a disadvantage on their carriers while offering long-term advantages to a population. The primary mechanistic solution to allow the evolution of far-sighted traits is a hierarchical structuring of the population-which includes group selection (Wynne-Edwards, 1962), kin selection (Hamilton, 1964), structured demes 
(Wilson, 1997), reciprocal altruism (Trivers, 1971), viscous populations (Hamilton, 1967; van Baalen and Rand, 1998; Mitteldorf et al., 2002a,b), 'multilevel' selection (Goodnight et al., 1992), 'lineage' selection (Nunney, 1999b), and metapopulation dynamics (Levins, 1968, 1970; McCauley, 1993).

Hierarchical structures that allow the evolution of far-sighted traits include a broad spectrum of possibilities. They may be discrete, such as a colony, isolated deme, or multicellular organism; they may be ephemeral, as in 'structured demes' (Wilson, 1977); or they may be continuous, such as viscous populations (Hamilton, 1967; Mitteldorf et al., 2002a,b). The requisite feature for all, however, is that the compositions of populations in different places be able to diverge from one another, so as to allow different fates to befall the different compositions.

\subsection{Evolutionary Stability through Evolvability Suppression}

The key theoretical concept to understanding when hierarchical dynamics can produce far-sighted traits is evolutionary stability (Maynard Smith and Price, 1973; Eshel and Feldman, 1982). Evolutionary stability poses two complementary questions:

1. Will genetic variation that enhances a far-sighted trait survive and flourish when introduced into a population?

2. Will genetic variation for a short-sighted trait (e.g. 'cheater' mutations) be driven to extinction when introduced into a population?

The existing theoretical solutions to the problem of far-sighted traits mainly take the evolutionary stability approach: conditions are found that prevent the invasion of shortsighted variants when they are introduced into a population, or prevent the extermination of far-sighted traits by short-sighted traits.

In the case when selection, genetic, and population parameters are in the right range, both conditions for the stability of far-sighted traits can be met. In the example above of longevity and parasite load, Kirchner and Roy (1999) find parameters in a metapopulation model that satisfy each of these evolutionary stability criteria, and prevent the evolution of longer life spans, reduce the parasite burden, and improve the population viability. Whether, or how often, these parameter conditions are met in nature continues to be a controversial issue (Goodnight and Stevens, 1997).

The evolutionary stability approach to the evolution of far-sighted traits contains within it a hidden presupposition: namely, that mutations that enhance or break down far-sighted traits-i.e. perturbations to the equilibria-occur with enough frequency to destabilize an unstable equilibrium. For example, the theory for the evolution of altruism assumes that 'cheater' mutations will arise with enough frequency to make the stability of altruistic traits an issue. The ability of a genome to generate variants in the direction of a certain trait is referred to as that trait's evolvability. Therefore, the evolutionary stability approach to understanding the evolution of far-sighted traits assumes the evolvability of these traits.

The possibility has been raised, however, the evolutionary stability of far-sighted traits against invasion by short-sighted traits need not always be a problem, because the 
evolvability of phenotypes that break down far-sighted traits may not necessarily exist. Let us take this possibility to the next level: suppose that evolution could find a way to prevent the generation of the short-sighted traits in the first place. This would provide another mechanism to stabilize far-sighted traits.

A number of studies in recent years have examined the question of how the evolvability of traits may itself evolve as a systematic outcome of evolutionary forces (Altenberg, 1985; Altenberg and Brutlag, 1986; Nunney, 1989; Altenberg, 1994a,b, 1995; Wagner and Altenberg, 1996; Wagner et al., 1999; Ancel and Fontana, 2000; Lipson et al., 2002; Gardner and Zuidema, 2003; Ofria et al., 2003; Toussaint, 2003). The focus of population genetics theory has traditionally been on the fate of genetic variation within populations, rather than on the processes that originate it. Levinton (1988, p. 494) states this succinctly:

Evolutionary biologists have been mainly concerned with the fate of variability in populations, not the generation of variability. ... This could stem from the dominance of population genetic thinking, or it may be due to a general ignorance of the mechanistic connections between the genes and the phenotype. Whatever the reason, the time has come to reemphasize the study of the origin of variation.

Suppose that the organism could be mutated so that it could no longer even generate the short-sighted traits. Would such mutations have a selective advantage? In other words, could genotypes with this suppressed evolvability of the short-sighted traits come to predominate in the population? Would it require hierarchical population dynamics? If so, this would be a novel, higher order evolutionary phenomenon made possible by hierarchical population dynamics. The suppression of evolvability would constitute a second-order form of evolutionary stability.

Wynne-Edwards (1964) was perhaps the first person to propose that the suppression of evolvability was a means to stabilize far-sighted traits. However, he did not propose any mechanism by which this could happen (Nunney, 1999b). Such a mechanism can be conceived as follows:

Suppose that there was some trait which would evolve by individual selection if variation for it existed, but which would increase the chance of the deme to go extinct (for example, the size variation in the smaller of the two competing species in Roughgarden's models (Roughgarden et al., 1983)). Now, suppose the variation between populations was for the rate of introduction of this phenotypic variant. This could be due to different pleiotropy, different population structure, different genetic systems, or mutation rates. These populations would last longer. Also, this could lock out imprudent predation, and overuse of resources. (Altenberg, 1984b)

The idea is that far-sighted traits could be stabilized by genetic changes that prevent 'cheats' from even being generated. The possibility that these evolvability-suppression mutations could be maintained by metapopulation dynamics (Altenberg, 1984a, reprinted in Appendix) was prompted by a model presented by Peck $(1984,1992)$ which showed that extinction and recolonization dynamics could maintain altruism. Metapopulation 
dynamics (Levins, 1968, 1970) refers to fragmented populations in which frequent local extinction and recolonization of demes occurs.

Nunney (1989) proposes the same mechanism to account for the maintenance of sex (a far-sighted trait) against invasion by asexual mutants (short-sighted traits). He analyzes a metapopulation model in which the rate at which asexual mutations arise is subject to variation. In his model, the rate of mutation to viable asexual phenotypes evolves to low values. Nunney (1999a) also analyzes a model of evolvability suppression against carcinogenesis, and finds that multiple loci could evolve to suppress the evolution of cancers.

In this paper, I wish to examine a concrete model for the evolution of evolvability suppressors that will allow for the evolution of multiple suppressor loci, modeling the mutation processes between all alleles, and including a spatial population structure to provide the hierarchical dynamics.

\section{The Model}

There has been debate about the definition of 'far-sighted' and 'short-sighted' traits, in particular, 'weak' and 'strong' altruism (Grafen, 1984; Wilson, 1990). Here I consider traits that are incontrovertibly 'short-sighted': traits which have a selective advantage which nevertheless systematically bring about their own long run extinction, and the extinction of the population that carries them (Hamilton, 1971). These would be the most extreme outcomes among the short-sighted traits listed above. When the process of 'survival of the fittest' results instead in extinction, I wish to call such a paradoxical outcome an 'evolutionary pathology'. The common property of evolutionary pathologies is that the trait under consideration gives its carriers a viability or reproductive advantage, and genetic variation for the trait tends to increase in frequency in the population; yet, once it becomes common, the trait has ecological or group properties that increase the rate of extinction of the population.

The basic question I want to answer here is, can the genome evolve so that it prevents pathological phenotypes from even being produced by mutation? There are two mechanisms by which mutations to the pathological phenotypes could be prevented. One is simply a lowered mutation rate for production of pathological alleles. The other is to require that multiple loci be mutated in order to express the pathological phenotype. There are many more possibilities that would involve the latter mechanism, so this is what I examine here. To investigate whether the evolvability of evolutionary pathologies can evolve, the following model is constructed. What the model wishes to test is whether the hierarchical population dynamics can cause the genotype to evolve to be multiple mutational steps away from the pathological trait.

The genotype of individuals consists of a primary locus and numerous modifier loci. In the primary locus, mutation of the wild type gives rise at low frequency to the pathological trait. Specifically, the case will be considered in which all but one of the alleles at the primary locus are wild type, and one is pathological, with uniform mutation between all alleles. A range of allele numbers are investigated, from 2 to 128 , 
to see how the evolvability of the pathology allele puts pressure on the modifier loci to evolve checkpoint alleles.

At the modifier loci, the wild-type alleles are permissive of expression of the pathological trait at the primary locus. A rare gain-of-function mutation of a modifier allele will produce an allele that blocks the expression of the pathological trait. The presence of the gain-of-function allele at any modifier locus blocks the expression of the pathological trait.

Because of their analogy to the 'checkpoint' genes in metazoan organisms that protect cells against evolution into cancer cells (Nojima, 1997; McKeon, 1999; Honda et al., 2004), I refer to these gain-of-function alleles as 'checkpoint positive' or ' $\mathrm{CHKPT}^{+}$', while the wild type will be called 'checkpoint negative' or CHKPT ${ }^{-}$. Specifically, the case will be considered in which 127 out of 128 alleles at the primary locus are wild type $\mathrm{CHKPT}^{-}$, and one out of the 128 alleles has the gain-of-function $\mathrm{CHKPT}^{+}$, with uniform mutation between all alleles. Thus under mutational pressure alone, only a small number of these 'checkpoint' alleles would ever be present in the population. If these alleles increase in frequency, under hierarchical population dynamics, to levels far from mutational equilibrium, it will demonstrate that checkpoints can evolve to block the introduction of evolutionary pathologies.

The population is divided up into a lattice of demes. Migration rates between demes is assumed to be lower than the frequency of extinctions of demes, so that migration does not result in a mixing of demes, but in recolonization.

The genetic structure is illustrated in Figure 1. All genotypes are selectively neutral except the one that expresses the pathological trait, which has a selective advantage within the deme. The population structure consists of a 16 by 16 array of demes (with periodic boundaries).

The demes are filled initially with genotypes that have a non-pathology allele at the primary locus, and permissive $\mathrm{CHKPT}^{-}$alleles at the modifier loci. Other parameters of the model are given in Table 1.

The population reproduces in discrete generations. Organisms are asexual. The Wright-Fisher model of multinomial sampling is used for reproduction: 2000 offspring for each deme are sampled i.i.d. (independently, identically distributed) from the offspring genotype distribution of the parent population of the deme. The offspring genotype distribution is generated under the assumptions that:

- mutation rates are equal for all loci;

- mutation rates are symmetric between all alleles; and

- each locus mutates independently of the others.

These 2000 offspring replace the 2000 parents in the deme.

The deme size remains constant unless the pathological trait appears. I assume that the pathological trait grows in frequency within the deme fast enough to be considered instantaneous relative to the other time scales in the model. Therefore, when a pathological trait appears in a deme, the deme goes extinct before the next iteration of migration, whereupon it is recolonized by a single individual from a random neighboring deme. 


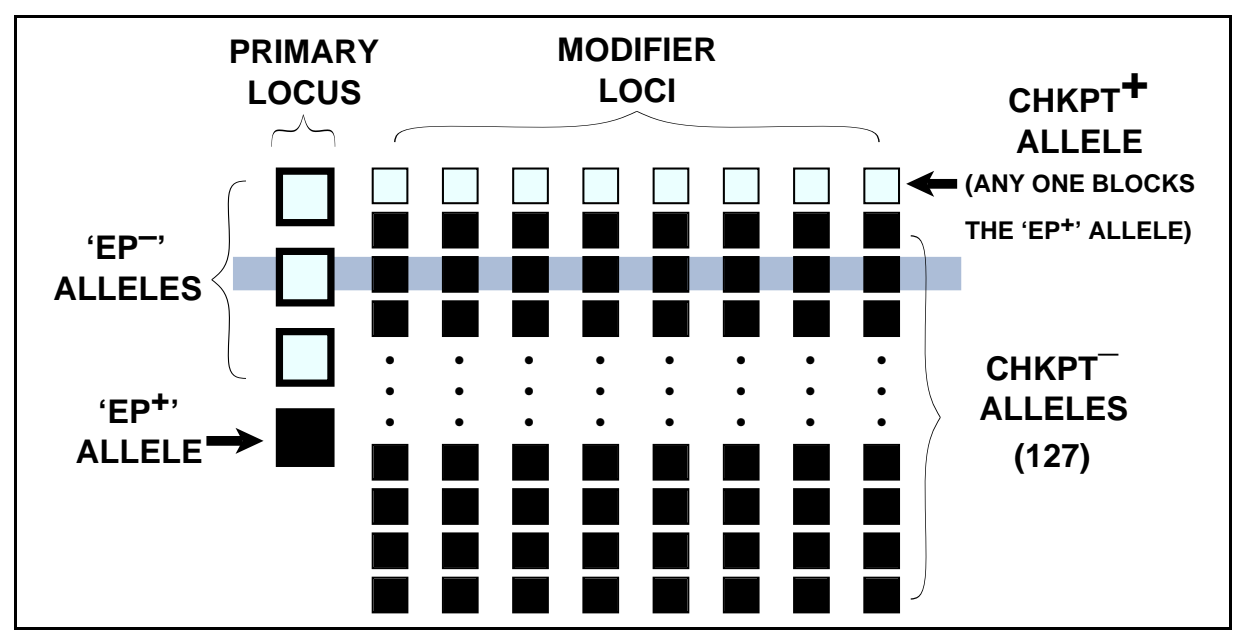

Figure 1: The genome in the evolvability suppression model. ' $\mathrm{EP}^{+}$' is the gain-offunction evolutionary pathology allele, ' $\mathrm{EP}^{-}$' is the wild-type, ' $\mathrm{CHKPT}^{+}$' is the gainof-function modifier allele that blocks expression of the pathological phenotype, and ' $\mathrm{CHKPT}^{-}$' is the permissive, wild-type modifier allele. 
- 1 primary locus, 8 modifier loci

- 2 to 128 alleles at the primary locus: $1 \mathrm{EP}^{+}, 1$ to $127 \mathrm{EP}^{-}$

- 128 alleles per modifier locus: $1 \mathrm{CHKPT}^{+}, 127 \mathrm{CHKPT}^{-}$

- 0.01 mutation rate per replication per locus

- $16 \times 16$ demes in the metapopulation

- 2000 individuals per deme

- Initial population: all individuals have the wild-type $\mathrm{EP}^{-}$primary gene and are all modifiers are $\mathrm{CHKPT}^{-}$

Table 1: Parameters of the model.

This model is designed to be as simple as possible, yet demonstrate the evolution of evolvability in the case of evolutionary pathologies. Clearly there are several avenues in which greater biological realism could be captured, with accompanying increases in the number of free parameters and dynamical variables. These elaborations include intra-deme dynamics of the pathological trait, specific ecological bases for the pathology, variation in the size of propagules during recolonization, sexual reproduction, recombination, and migration. Also, the model could be extended to include modifier variation that is not neutral. These elaborations are deferred to treatments more extensive than the present work.

\subsection{Model Details}

For concreteness it will be helpful to describe the model mathematically. Let

$N$ be the population size of each deme,

$A_{P}$ be the number of alleles at the primary locus (only one of which is the pathological allele, $\mathrm{EP}^{+}$), 
$A_{M}$ be the number of alleles at each modifier locus (only one of which is the $\mathrm{CHKPT}^{+}$ allele)

$L$ be the number of modifier loci, and

$\mu$ be the mutation rate per locus per generation.

I assume a uniform mutation model, in which all transitions between alleles are equally likely. Hence this is a 'House of Cards' model (Kingman, 1978)(Kingman, 1980, p. 15). This symmetry allows us to lump all the non-pathological alleles $\mathrm{EP}^{-}$into a single class, indexed with 1 , while the pathological allele $\mathrm{EP}^{+}$is indexed with 0 . Then the transition probabilities under mutation for alleles at the primary locus are:

$$
\begin{aligned}
& \operatorname{Prob}[0 \leftarrow 1]=p_{01}=\mu\left(\frac{1}{A_{P}}\right) \text { and } \\
& \operatorname{Prob}[1 \leftarrow 0]=p_{10}=\mu\left(\frac{A_{P}-1}{A_{P}}\right)=\mu\left(1-\frac{1}{A_{P}}\right) .
\end{aligned}
$$

The symmetry also allows us to lump all the $\mathrm{CHKPT}^{-}$alleles into a single class, indexed with 0 , while the $\mathrm{CHKPT}^{+}$allele is indexed with 1 (this choice is made so that the pathological phenotype is expressed in the genotype of all 0s). The transition probabilities under mutation for alleles at each modifier locus are:

$$
\begin{aligned}
& \operatorname{Prob}[0 \leftarrow 1]=m_{01}=\mu\left(\frac{A_{M}-1}{A_{M}}\right)=\mu\left(1-\frac{1}{A_{M}}\right) \text { and } \\
& \operatorname{Prob}[1 \leftarrow 0]=m_{10}=\mu\left(\frac{1}{A_{M}}\right) .
\end{aligned}
$$

These probabilities give the following transition matrices for mutation at the primary and modifier loci:

$$
\boldsymbol{P}=(1-\mu) \boldsymbol{I}+\mu\left[\begin{array}{cc}
\frac{1}{A_{P}} & \frac{1}{A_{P}} \\
1-\frac{1}{A_{P}} & 1-\frac{1}{A_{P}}
\end{array}\right]
$$

and

$$
\boldsymbol{M}=(1-\mu) \boldsymbol{I}+\mu\left[\begin{array}{cc}
1-\frac{1}{A_{M}} & 1-\frac{1}{A_{M}} \\
\frac{1}{A_{M}} & \frac{1}{A_{M}}
\end{array}\right]
$$

With the assumption that mutation occurs independently at each locus, the transition probability matrix for the entire genotype is:

$$
\boldsymbol{T}=\boldsymbol{P} \otimes \boldsymbol{M}^{\otimes L},
$$

where $\otimes L$ represents $L$ tensor products of $M$.

An alternative representation of the state space for the modifier loci is to lump all the modifier loci together, and simply count the number of $\mathrm{CHKPT}^{+}$alleles among them. To get a transition from $j$ to $i \mathrm{CHKPT}^{+}$alleles, mutation must produce a net change of $i-j$ in the $\mathrm{CHKPT}^{+}$count. We calculate this be summing over all possible 
ways to get this net change, i.e. all the values of $k$, where $k \mathrm{CHKPT}^{+}$alleles mutate to $\mathrm{CHKPT}^{-}$, and $i-j+k \mathrm{CHKPT}^{-}$alleles mutate to $\mathrm{CHKPT}^{+}$:

$$
\begin{aligned}
\tilde{m}_{i j}=\sum_{k=0}^{\min (i, j, L-i, L-j)} & \left(\begin{array}{c}
j \\
k+\phi(j, i)
\end{array}\right) m_{01}^{k+\phi(j, i)}\left(1-m_{01}\right)^{j-k-\phi(j, i)} \\
& \left(\begin{array}{c}
L-j \\
k+\phi(i, j)
\end{array}\right) m_{10}^{k+\phi(i, j)}\left(1-m_{10}\right)^{L-j-k-\phi(i, j)}
\end{aligned}
$$

where $\phi(i, j)=\max (i-j, 0)$. Clearly (2) defines a Markov chain, but its terms are not easily digested. Form (2) is useful nevertheless for a numerical implementation of the model, because the values $\tilde{m}_{i j}$ need be calculated only once, and then a single call to a random number generator is sufficient to determine the transition. Direct implementation of (1) would require $L$ random number calls per transition, which is computationally more costly with large population sizes and numbers of generations.

For small mutation rates, (2) is approximated as:

$$
\tilde{m}_{i j}=\left\{\begin{array}{cl}
(L-j) m_{10} & \text { if } i=j+1 \\
1-j m_{01}-(L-j) m_{10} & \text { if } i=j \\
j m_{01} & \text { if } i=j-1
\end{array}\right.
$$

To complete the description of the evolution within a deme we include WrightFisher multinomial sampling. The elements of the Wright-Fisher model are as follows:

$\boldsymbol{x}$ is the vector of frequencies of each genotype $i$ in the population, corresponding to $N x_{i}$ individuals of genotype $i$;

$\boldsymbol{y}=\boldsymbol{T} \boldsymbol{x}$ is the vector representing the probability distribution for sampling a genotype among the offspring produced by population $\boldsymbol{x}$;

$\boldsymbol{x}^{\prime}$ is the vector of the frequencies of each genotype $i$ in the population in the next generation, corresponding to $N x_{i}^{\prime}$ individuals of genotype $i$, produced by taking $N$ independent samples from the distribution $\boldsymbol{y}(\boldsymbol{x})$;

$n=2^{L+1}$ be the number of possible genotypes.

Since the population consists of discrete individuals, the frequency vectors are now restricted to a lattice of discrete points on the simplex $\Delta_{n}$, namely

$$
\Delta_{n}(N)=\left\{\boldsymbol{x}: N x_{i} \in\{0,1, \ldots, N\}, \sum_{i=1}^{n} x_{i}=1\right\} .
$$

The Wright-Fisher model forms a Markov chain, whose transition matrix on frequency vectors is:

$$
\boldsymbol{G}=\left[G_{\boldsymbol{x}^{\prime}, \boldsymbol{x}}\right]_{\boldsymbol{x}, \boldsymbol{x}^{\prime} \in \Delta_{n}(N)}
$$

with entries

$$
G \boldsymbol{x}^{\prime}, \boldsymbol{x}=N ! \prod_{i=1}^{n} \frac{y_{i}^{N x_{i}^{\prime}}}{\left(N x_{i}^{\prime}\right) !}=\frac{N !}{\prod_{i=1}^{n}\left(N x_{i}^{\prime}\right) !} \prod_{i=1}^{n}\left(\boldsymbol{e}_{i}^{\top} \boldsymbol{T} \boldsymbol{x}\right)^{N x_{i}^{\prime}}
$$


where $\boldsymbol{e}_{i}^{\top}=\left[\begin{array}{llllll}0 & 0 & \cdots & 1 & \cdots & 0\end{array}\right]$ has the 1 in the $i$ th position.

The probability distribution on population compositions as a function of generation time $t$ is thus:

$$
\boldsymbol{\xi}(t)=\boldsymbol{G}^{t} \boldsymbol{e}_{k}
$$

where $\xi(t)$ is the vector of probabilities for different population compositions at time $t$, and $k$ is the genotype that recolonized the deme after its last extinction.

A full analysis of (5) is not necessary to see what is happening in the model. In this model, a deme goes extinct as soon as it generates its first pathological phenotype. Since demes are founded by a single individual, all the genotypes are initially the same, and non-pathological. The model is designed so that mutation pressure alone within a population will steadily reduce the number of $\mathrm{CHKPT}^{+}$alleles. When mutation pressure has knocked out all the $\mathrm{CHKPT}^{+}$alleles, then the appearance of the pathology allele at the primary locus will produce the pathological phenotype. As time goes on, the probability that one of the individuals will have mutated to the pathological phenotype increases monotonically. Demes with a greater number of $\mathrm{CHKPT}^{+}$alleles will have a greater longevity, and thus be more likely to be the source of propagules to recolonize extinct demes. Through this differential viability of demes, increased numbers of $\mathrm{CHKPT}^{+}$alleles in the genome can evolve.

\section{Results}

The trajectories of a typical runs of this model is shown in Figure 2 for the case of $1 \mathrm{EP}^{+}$and $3 \mathrm{EP}^{-}$alleles at the primary locus. It shows the distribution of the mean number of $\mathrm{CHKPT}^{+}$alleles per individual in each deme as evolution progresses.

The distribution of the metapopulation is initialized at generation 0 to fall along on the X-axis, i.e. all demes have an average of zero $\mathrm{CHKPT}^{+}$alleles per individual. Under mutation pressure alone, the population would equilibrate to the dotted line at $0.0625=1 / 16=8$ loci $/ 128$ alleles per locus. After 100 generations of extinction and recolonization dynamics, however, almost all demes have an average of one $\mathrm{CHKPT}^{+}$ allele per individual. By generation 200, most demes average two $\mathrm{CHKPT}^{+}$alleles per individual, with a few demes having more or fewer.

As the generations continue, demes averaging just under $3 \mathrm{CHKPT}^{+}$alleles increase to around half of the total number. A fraction of the demes average as many as 4 $\mathrm{CHKPT}^{+}$alleles per individual. The consequence of having multiple $\mathrm{CHKPT}^{+}$loci is that there is little likelihood that a deme will generate an individual with the pathological trait. Hence, the evolvability of the pathological trait has been suppressed.

It is notable that the distributions are concentrated around integral numbers of $\mathrm{CHKPT}^{+}$alleles. This occurs because each deme is recolonized by a single individual with a discrete number of $\mathrm{CHKPT}^{+}$alleles.

As mutations accumulate, the distribution shifts downward toward the equilibrium line at 0.0625 . Once a deme is recolonized, it 'ages' with the mutational loss of $\mathrm{CHKPT}^{+}$alleles. There is no dynamic to increase the number of $\mathrm{CHKPT}^{+}$alleles above the mutational equilibrium frequency of 0.0625 in an existing deme. 


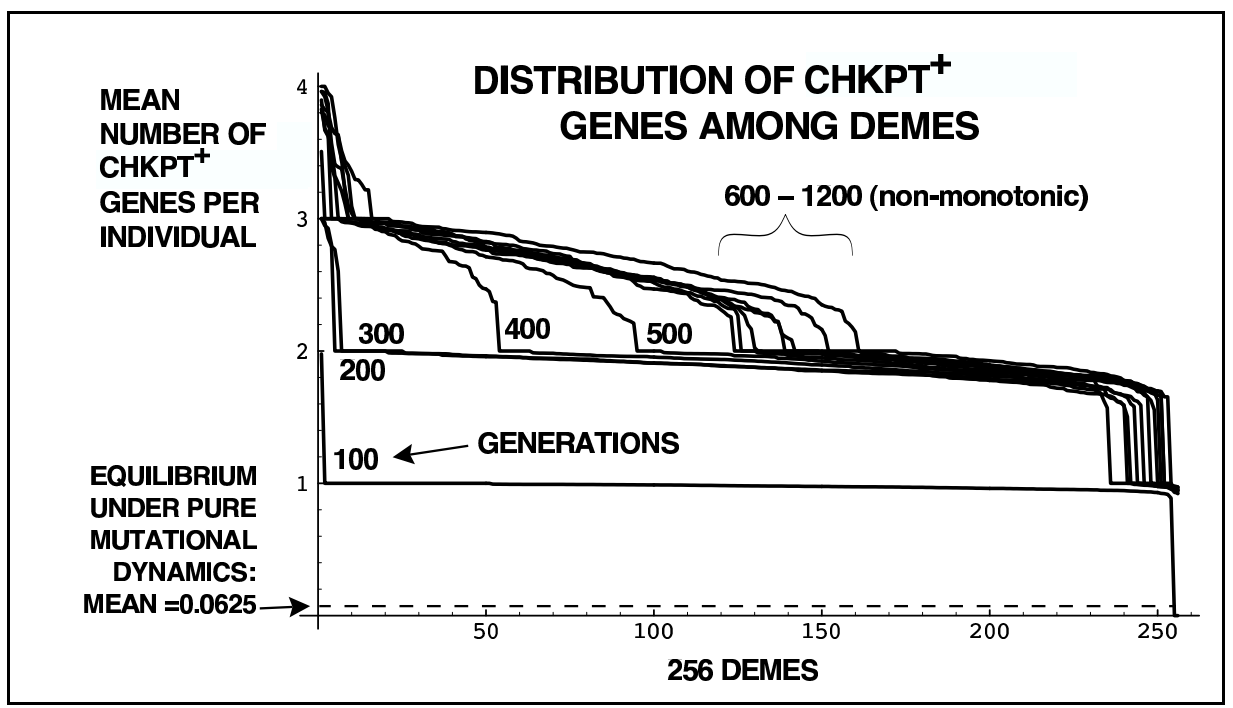

Figure 2: Evolution of evolvability suppression. The mean number of $\mathrm{CHKPT}^{+}$alleles

per individual in each deme is calculated, and the demes sorted by this value along

the $\mathrm{X}$-axis, and the value plotted. In this run there are $1 \mathrm{EP}^{+}$and $3 \mathrm{EP}^{-}$alleles at the

primary locus.

As demes age and more individuals lose their $\mathrm{CHKPT}^{+}$alleles, the chance of generating the pathological trait increases and, along with that, deme extinction. Hence, deme extinctions are drawn predominantly from older demes, while deme recolonizations are a uniform sample of individuals in the deme neighborhood. The difference in the distributions of extinctions and recolonizations leads to the increase in the number of $\mathrm{CHKPT}^{+}$alleles per individual over the metapopulation.

The effect of allele number at the primary locus, $A_{P}$, can be seen in Figure 3. Under the house-of-cards mutation model, the rate of mutation to the $\mathrm{EP}^{+}$allele is $p_{01}=\mu / A_{P}$. Hence, $p_{01}$ is a measure of the baseline evolvability of the pathological trait in the absence of any $\mathrm{CHKPT}^{+}$evolvability suppressors. We would expect that the higher the baseline evolvability of the pathological trait is, the greater the pressure for its suppression should be. This is confirmed in Figure 3.

In this figure, the population is always censused at generation 1000, at which time the steady-state has been attained. The different distributions represent the outcome for a range of allele numbers, $A_{P}$, at the primary locus. For the minimum number of $A_{P}=2$, this means that half of all mutations at the primary locus will be to the pathological $\mathrm{EP}^{+}$allele. For the maximum number of $A_{P}=128$ alleles, only $1 / 128$ of mutations will produce the pathological $\mathrm{EP}^{+}$allele. The system can respond by tuning 
the number of $\mathrm{CHKPT}^{+}$alleles to the evolvability of $\mathrm{EP}^{+}$.

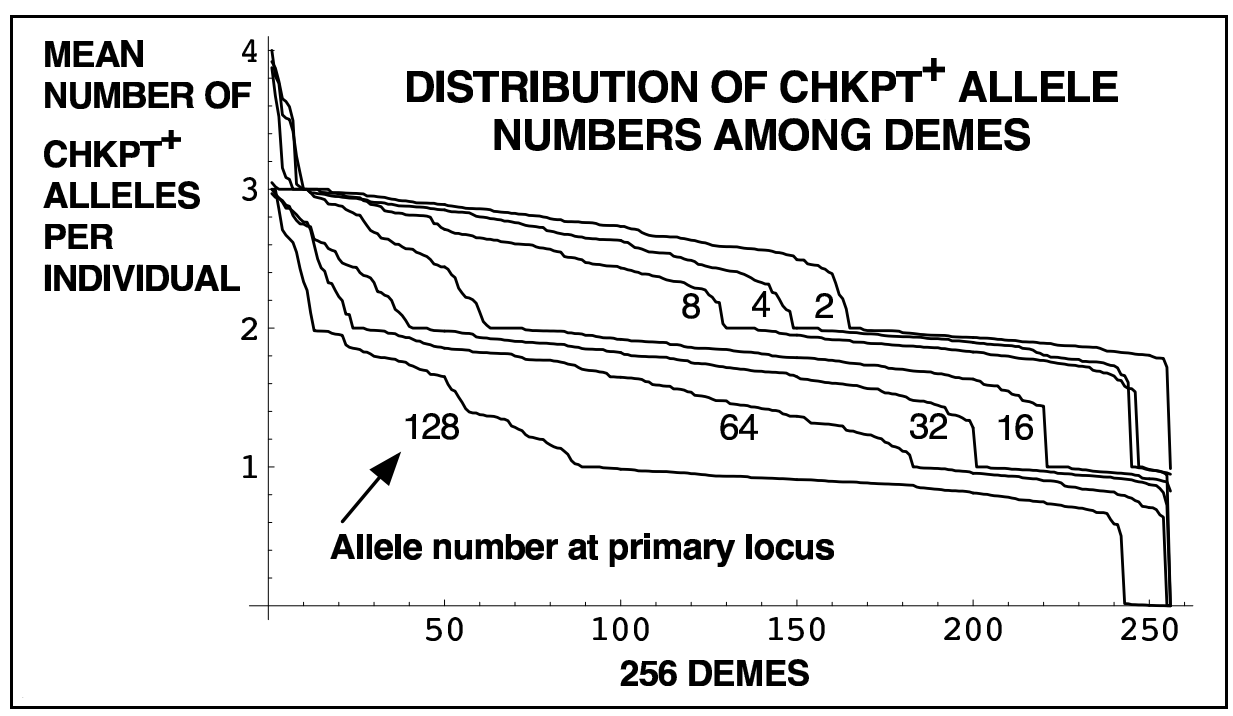

Figure 3: Evolvability suppression as a function of mutation pressure. Distributions

in the mean number of $\mathrm{CHKPT}^{+}$alleles per individual in each deme are shown as a function of the number of alleles at the primary locus, ranging from 2 to 128 . At the primary locus there is always $1 \mathrm{EP}^{+}$allele, and a range of between 1 to $127 \mathrm{EP}^{-}$ alleles. Census is at generation 1000 . The greater the mutation pressure to the $\mathrm{EP}^{+}$ allele (fewer $\mathrm{EP}^{-}$alleles), the greater the number of $\mathrm{CHKPT}^{+}$alleles that evolve to suppress the pathology phenotype.

This is what is observed. For $A_{P}=2,4$, and 8 , some fraction of demes is found with mean numbers of $\mathrm{CHKPT}^{+}$alleles greater than 3 . For $A_{P}=16$ and higher, some demes are always found with mean numbers of $\mathrm{CHKPT}^{+}$alleles greater than 2 . Only when $A_{P}=128$ can any demes be found that have no $\mathrm{CHKPT}^{+}$alleles.

Thus, the higher the baseline evolvability is for the pathological trait, the greater is the number of evolvability suppressors that will be maintained by extinction and recolonization dynamics. 


\section{Discussion}

The possibility that hierarchically structured populations can affect the evolvability of short-sighted traits (evolutionary pathologies) has been verified in this simple concrete model. Previous work (Nunney, 1985) has shown that hierarchically structured populations can lead to the survival of far-sighted traits, and the suppression of short-sighted traits, when genes for them are introduced into a population. Here, we find that even the process which introduces short-sighted traits can itself come under selection in hierarchically structured populations, and be caused to suppress the very generation of evolutionary pathologies by mutation.

\subsection{Evolvability modifiers or simply epistatic loci?}

It might be asked whether the 'checkpoint' genes modeled here (Figure 1) are really evolvability modifiers, or rather simply genes that epistatically interact with the primary locus to control the pathology phenotype. Indeed, the only distinction between the primary locus and the modifier loci is in the asymmetry between the number of alleles that allow the pathological trait to be expressed: in the primary locus, 1 out of $A_{P}$ alleles $\left(A_{P} \in\{2,4,8,16,32,64,128\}\right)$ allow expression of the pathological trait; in the modifier loci, 127 out of 128 alleles allow the expression. Loss of the pathological trait is the same whether due to a mutation at the primary locus, or a mutation at one modifier locus to $\mathrm{CHKPT}^{+}$.

Thus, one might argue that the results seen here do not constitute an evolution of evolvability, but are simply metapopulation selection against the pathological trait itself, as seen in Kirchner and Roy (1999). This hypothesis, however, would explain the evolution of at most one $\mathrm{CHKPT}^{+}$locus. The evolution of additional $\mathrm{CHKPT}^{+}$loci has no effect on the phenotype, but rather, affects only the rate at which pathological variants arise by mutation-i.e. the evolvability of the pathological trait (Altenberg, 1995). We observe in the simulation that the metapopulation evolves to the point where most demes average from 2 up to $4 \mathrm{CHKPT}^{+}$loci per individual for small values of $A_{P}$.

Nunney (1999a) investigates a related model of multistep carcinogenesis and finds conditions that would support the evolution multiple evolvability suppressor loci. The model differs in a number of ways from the present study: instead of a Wright-Fisher model of finite populations, it models cells in a tissue that divide until a certain number is reached. This model is readily analyzed, and when the cell number and mutation rate are high enough, multiple tumor suppressor loci are required to maintain at least one of them in all cells.

The present model is oriented toward multicellular organisms that reproduce in a spatial structure, with extinction and recolonization dynamics. While the model makes for a more protracted analysis, the numerical simulation of the dynamics reveals details of the steady-state distribution of the number of evolvability suppressor alleles. The overall result parallels the results of Nunney (1999a): strong mutation pressure toward $\mathrm{CHKPT}^{-}$alleles is overcome by the metapopulation dynamics. The primary consequence is that evolvability of the evolutionary pathology is suppressed, and the rate of deme extinction is reduced because of evolution at the modifier loci. 


\subsection{Evolvability suppression and canalization}

The dynamics of this model is closely related to that of neutral network models of canalization. The concept of canalization was proposed by Schmalhausen (1949, pp. 94-95) and Waddington (1942). Canalization is defined as the accumulation of mutations that stabilize a phenotype against either genetic or environmental perturbations. The CHKPT $^{+}$loci stabilize the non-pathological trait against mutations.

The usual condition that promotes canalization is stabilizing selection, when departures from the phenotype are deleterious (canalization thus lowers the mutational load). In the case of evolutionary pathologies, however, instead of stabilizing selection against the pathology phenotype, there is directional selection in favor of it. Only the presence of metapopulation dynamics prevents the immediately advantageous pathology phenotype from fixing in the population. Nevertheless, the metapopulation dynamics has the same long term effect that stabilizing selection would were it acting against the pathological trait.

\subsection{Neutral network dynamics in the model}

Since all the different genotypes containing $\mathrm{CHKPT}^{+}$alleles or $\mathrm{EP}^{-}$alleles have equal fitness, they comprise a neutral network under stabilizing selection (Fontana et al., 1993). The population is forced by metapopulation dynamics to remain on this neutral network, since as soon as a genotype mutates off of the network to the pathology phenotype, its deme goes extinct.

In their study of evolution on neutral networks, van Nimwegen et al. (1999) emphasize that "a population seeks out the most connected areas of the neutral network". By "the most connected areas", they mean genotypes that produce a large number of neutral mutations, since neutral mutations are what counts as a connections in the neutral network graph.

However, we see in this model that the feature that the population seeks is not "highly connected areas of the network", but rather genotypes that are further from the "edge" of the network. The "connectedness" of genotypes are all approximately the same when they have 2, 3, 4, and more $\mathrm{CHKPT}^{+}$alleles, in that all of their singlemutant neighbors are still on the network (the double and higher mutations are so rare for small mutation rates that they have little influence). The difference between them is their distance from the edge of the network, the edge being the genotypes with no $\mathrm{CHKPT}^{+}$alleles. The number of $\mathrm{CHKPT}^{+}$loci equals the mutational distance from the edge of the neutral network. Populations are initialized on the edge of the network, and evolve by moving away from the edge. The stronger the baseline evolvability of the pathological trait, the further away from the edge they evolve.

Thus, this model demonstrates an important principle for understanding evolution on neutral networks under stabilizing selection: is not the local connectedness of regions in the network that make them attractors, but rather their distance from the boundaries of the network, which is a global feature of network structure. 


\subsection{The necessity for single-organism propagules}

A crucial element of the model that is necessary for $\mathrm{CHKPT}^{+}$loci to evolve is that demes be recolonized through extreme population bottlenecks. If recolonized demes were simple copies of a neighboring deme in their genotypes distribution, there would be no way to reverse the mutational pressure towards lower values of $\mathrm{CHKPT}^{+}$loci. Very strong genetic drift is required to produce occasionally higher numbers of $\mathrm{CHKPT}^{+}$ loci, and the model here maximizes the force of drift by recolonizing with a single individual. Clearly, the magnitude of drift effects will be decreased by propagules with larger numbers of individuals, producing fewer demes with extreme numbers of $\mathrm{CHKPT}^{+}$loci. Consequently, large propagules will hinder the evolution of $\mathrm{CHKPT}^{+}$ loci.

It is notable that multicellular organisms almost universally start as single individual cells. Michod and Roze (1999) point out that this prevents the spread of mutants that defect from multicellular cooperation. When the multicellular organism is taken as the deme, and its death as the extinction of the deme, then the dynamics can be seen to be homologous.

\subsection{Plastid gene migration to the nucleus: driven by evolvability}

\section{suppression?}

Evolutionary pathologies can be prophylactically prevented through evolvability suppression, which is the elimination of heritable variation for the pathology at the lowerlevel of the population hierarchy. The model of modifiers genes considered here is but one means by which the generation of heritable variation may be suppressed. In this modifier gene model, the mechanism of control is distributed among the lowerlevel individuals in the population hierarchy, and acts locally within the individuals. Replication and mutation of the lower-level individuals occurs independently of one another. The only structures that create dependence between lower-level individuals is their common descent from the single recolonizer of their deme, the cloistering of reproduction within the demes, and the extinction of demes.

If the independence of replication could be taken away from the lower-level individuals, then heritable variation for the pathological trait and natural selection between them could be prevented. If all individuals at the lower-level were compelled to share the same phenotype, then no selective differences could arise. A means to achieve this would be to remove the pathology gene from the lower-level individuals, and move its function to their common environment. This comprises an alternative to modifier genes for evolvability suppression.

One example of this particular phenomenon comes to mind: the transfer of genes from mitochondria and chloroplasts to the nucleus. Mitochondria and chloroplasts correspond to lower-level, endosymbiotic individuals replicating within a deme, the eukaryotic cell. The cell has its own 'deme-level' inheritance mechanism, the nucleus.

The dynamics underlying the migration of genes from endosymbionts to the host genome—a systematically repeated phenomenon—are not resolved. Berg and Kurland 
(2000) investigate whether differential mutation rates can produce the gene migration. The possibility I wish to propose here is that the migration of plastid genes to the nucleus is favored because it is a form of evolvability suppression.

A plastid gene that mutates to increase plastid proliferation, at the expense of cell function, is an evolutionary pathology. If such a gene has migrated to the nucleus, all the plastids will share its phenotype, so heritable variation and differential replication of plastids cannot occur. Hence, only mutations that are beneficial to the cell-the higher level in the hierarchy - will be selected. Thus, plastid genes that migrate into the nucleus are no longer vulnerable to evolutionary pathologies. Furthermore, cellular mechanisms promoting the transfer of plastid genes to the nucleus would be secondarily selected. Thus, evolvability suppression is a hypothesis that may account for the migration of plastid genes to the nucleus.

\subsection{The emergence of evolvability suppressors}

The model considered here makes a clear distinction between the primary locus and the evolvability modifier loci. In real organisms however, as well as in the most interesting artificial life systems, genes have emergent functions that are not pre-labeled as 'primary' or 'modifier' loci. Similarly, the phenotypes are not pre-labeled as 'far-sighted', 'short-sighted', or 'pathological', but their effects on the short or long term survival of their carriers are emergent properties. These properties may not be inferable from a reductionist description of them. Hence, real organisms and artificial life systems may exhibit evolutionary pathologies as emergent phenomena that can only be observed retrospectively.

Mechanisms that can suppress evolvability may also be emergent. One adaptation may close off the pathway to another adaptation. For example, the evolution of a large mouth could preclude the capturing prey in crevices. Evolutionary tradeoffs and developmental constraints are ubiquitous possibilities.

What the present study shows is that a trait which has the happenstance ability to foreclose pathways to evolutionary pathologies may evolve for that reason, and not for what may appear to be its obvious 'functions'.

Mitotic checkpoint genes, which may have originally evolved to ensure viable mitosis, may have been exapted (Gould and Vrba, 1982) to ensure the inviability of defective mitosis in higher organisms.

The suppression of evolvability for evolutionary pathologies becomes a possibility in evolutionary systems that contain structured populations. Structured populations abound in biological systems, and have been investigated in evolutionary computation and artificial life as well (D'haeseleer and Bluming, 1994; Ray, 1995).

Because of the complexity of organismal phenotypes, the effect of one trait on another trait's evolvability may not be obvious, or even in principle predictable. Hence, structured populations may contain adaptations to suppress evolutionary pathologies where there is no way for the observer to know that this is their purpose. However, if Nature, or the experimenter, changes the population structure from multiple demes to a single interacting population, the suppression of evolutionary pathologies may become 
known by observing its breakdown: genetic variability for pathological traits that had been suppressed under a structured population could be expected to emerge in a newly panmictic population. In biological and artificial life systems where phenotypes and evolutionary dynamics are emergent, the best methodology to explore the suppression of the evolvability of evolutionary pathologies may be through experimental alterations in population structure.

\subsection{Conclusion}

The model examined here demonstrates another circumstance in which evolvability can evolve. What evolves here is not an organismal phenotype, but the genome's propensity to generate phenotypic variants. In the present case, when the trait confers short-term individual advantage but long-term population disadvantage, under a hierarchically structured population, evolvability evolves to be suppressed. The net effect of these hierarchical evolutionary dynamics is, once again, to increase the survival of the organism.

\section{Acknowledgements}

Thanks to the Santa Fe Institute, the Evolvability Working Group, Tom Ray and his research grant from the Grateful Dead, the Keck Foundation for inviting this work and funding the collegial interaction that facilitated it, and to the editors of this special issue of Artificial Life, Richard Watson and Dominique Chu.

\section{Bibliography}

Altenberg, L. 1984. Group selection model for limitation of group disadvantageous mutation. Research Notes, Evolutionary Lock Out, entry August 4, 1984.

Altenberg, L. 1984. Locking-out prisoner's dilemma genes. Research Notes, Evolutionary Lock Out, 1984.

Altenberg, L. 1985. Knowledge representation in the genome: new genes, exons, and pleiotropy. Genetics, 110, supplement:s41. Abstract of paper presented at the 1985 Meeting of the Genetics Society of America.

Altenberg, L. 1994. The evolution of evolvability in genetic programming. In Kinnear, K. E., editor, Advances in Genetic Programming, pages 47-74. MIT Press, Cambridge, MA.

Altenberg, L. 1994. Evolving better representations through selective genome growth. 
In Proceedings of the 1st IEEE Conference on Evolutionary Computation. Part 1 (of 2), pages 182-187, Piscataway N.J. IEEE.

Altenberg, L. 1995. Genome growth and the evolution of the genotype-phenotype map. In Banzhaf, W. and F. H. Eeckman, editors, Evolution and Biocomputation: Computational Models of Evolution, volume Lecture Notes in Computer Science 899, pages 205-259. Springer-Verlag, Berlin.

Altenberg, L. and D. L. Brutlag. 1986. Selection for modularity in the genome. Unpublished. Cited in Doolittle (1987), and Tomita et al. (1996).

Ancel, L. W. and W. Fontana. 2000. Plasticity, evolvability and modularity in RNA. Journal of Experimental Zoology (Molecular and Developmental Evolution), 288:242283.

Berg, O. G. and C. G. Kurland. 2000. Why mitochondrial genes are most often found in nuclei. Molecular Biology and Evolution, 17(6):951-961.

D'haeseleer, P. and J. Bluming. 1994. Effects of locality in individual and population evolution. In Kinnear, Jr., K. E., editor, Advances in Genetic Programming, pages 177-198. MIT Press, Cambridge, MA.

Doolittle, W. F. 1987. The origin and function of intervening sequences in DNA: A review. American Naturalist, 130:915-928.

Eshel, I. and M. W. Feldman. 1982. On the evolutionary stability of the sex ratio. Theoretical Population Biology, 21:430-439.

Fontana, W., P. F. Stadler, E. G. Bornberg-Bauer, T. Griesmacher, I. L. Hofacker, M. Tacker, P. Tarazona, E. D. Weinberger, and P. Schuster. 1993. RNA folding and combinatory landscapes. Physical Review E, 47(3):2083-2099.

Gardner, A. and W. Zuidema. 2003. Is evolvability involved in the origin of modular variation? Evolution, 57:1448-1450.

Goodnight, C. J., J. M. Schwartz, and L. Stevens. 1992. Contextual analysis of models of group selection, softselection, hard selection, and the evolution of altruism. American Naturalist, 140:743-761.

Goodnight, C. J. and L. Stevens. 1997. Experimental studies of group selection: what do they tell us about group selection in nature? The American Naturalist, 150, Supplement:S59-S79.

Gould, S. J. and E. S. Vrba. 1982. Exaptation: A missing term in the science of form. Paleobiology, 8(1):4-15.

Grafen, A. 1984. Natural selection, group selection, and kin selection. In Behavioural Ecology, chapter 3, page 6280. Blackwell, London, 2nd edition. 
Griffiths, H. I. and R. K. Butlin. 1995. A timescale for sex versus parthenogenesis: Evidence from subfossil ostracods. Proceedings of the Royal Society of London Series B: Biological Sciences, 260(1357):65-71.

Hamilton, W. D. 1964. The genetical evolution of social behaviour. I. Journal of Theoretical Biology, 7:1-16.

Hamilton, W. D. 1967. Extraordinary sex ratios. Science, 156:477-488.

Hamilton, W. D. 1970. Selfish and spiteful behaviour in an evolutionary model. Nature, 228:1218-1220.

Hamilton, W. D. 1971. Selection of selfish and altruistic behaviour in some extreme models. In Eisenberg, J. F. and W. S. Dillon, editors, Man and Beast: Comparative Social Behavior, pages 57-91. Smithsonian Press, Washington, DC.

Hardin, G. 1968. The tragedy of the commons. Science, 162:1243-1248.

Honda, T., G. Tamura, T. Waki, S. Kawata, S. Nishizuka, and T. Motoyama. 2004. Promoter hypermethylation of the chfr gene in neoplastic and non-neoplastic gastric epithelia. British Journal of Cancer, 90:2013-2016.

Kauffman, S. A. and S. Johnsen. 1991. Coevolution to the edge of chaos: Coupled fitness landscapes, poised states, and coevolutionary avalanches. Journal of Theoretical Biology, 149(4):467-506.

Kingman, J. F. C. 1978. A simple model for the balance between selection and mutation. Journal of Applied Probability, 15:1-12.

Kingman, J. F. C. 1980. Mathematics of Genetic Diversity. Society for Industrial and Applied Mathematics, Philadelphia. ISBN 0-89871-166-5.

Kirchner, J. W. and B. A. Roy. 1999. The evolutionary advantages of dying young: Epidemiological implications of longevity in metapopulations. American Naturalist, 154(2):140-159.

Levins, R. 1968. Evolution in Changing Environments. Monographs in Population Biology 2. Princeton University Press, Princeton, New Jersey.

Levins, R. 1970. Extinction: some mathematical questions in biology. In Gerstenhaber, M., editor, Lectures on Mathematics in the Life Sciences, volume 2, pages 75108. American Mathematical Society, Providence, Rhode Island.

Levinton, J. 1988. Genetics, Paleontology, and Macroevolution. Cambridge University Press, Cambridge.

Lewontin, R. C. 1962. Interdeme selection controlling a polymorphism in the house mouse. American Naturalist, 96:65-78. 
Lipson, H., J. B. Pollack, and N. P. Suh. 2002. On the origin of modular variation. Evolution, 56:1549-1556.

Maynard Smith, J. 1964. Group selection and kin selection. Nature, 201:1145-1147.

Maynard Smith, J. and G. R. Price. 1973. The logic of animal conflict. Nature, 246: $15-18$.

McCauley, D. E. 1993. Evolution in metapopulations with frequent local extinction and recolonization. In Futuyma, D. and J. Antonovics, editors, Oxford Surveys in Evolutionary Biology, volume 9, pages 110-134. Oxford University Press, Oxford.

McKeon, F. 1999. Killing the umpire: cooperative defects in mitotic checkpoint and brca2 genes on the road to transformation. Breast Cancer Research, 1:8-10.

Michod, R. E. and D. Roze. 1999. Cooperation and conflict in the evolution of individuality. III. Transitions in the unit of fitness. In Nehaniv, C. L., editor, Lectures on Mathematics in the Life Sciences, volume 26, pages 47-91. American Mathematical Society, Providence, Rhode Island.

Mitteldorf, J., D. H. Croll, and S. C. Ravela. 2002. Multilevel selection and the evolution of predatory restraint. In Standish, R., M. A. Bedau, and H. A. Abbass, editors, Artificial Life VIII: Proceedings of the Eighth International Conference on Artificial Life, pages 146-152, Cambridge, Massachusetts. The MIT Press. ISBN 0-262-692813.

Mitteldorf, J., S. Ravela, D. H. Croll, R. Bell, D. Seetharam, and D. Boccelli. 2002. On the prudent predator: Individual-based modeling of multilevel selection and the evolution of predatory restraint. InterJournal Complex Systems, Online:MS no. 540.

Nojima, H. 1997. Cell cycle checkpoints, chromosome stability and the progression of cancer. Hum. Cell, 10(4):221-230.

Nunney, L. 1985. Group selection, altruism, and structured-deme models. American Naturalist, 126:212-230.

Nunney, L. 1989. The maintenance of sex by group selection. Evolution, 43(2):245257.

Nunney, L. 1999. Lineage selection and the evolution of multistage carcinogenesis. Proceedings of the Royal Society of London Series B: Biological Sciences, 266:493498.

Nunney, L. 1999. Lineage selection: Natural selection for long-term benefit. In Keller, L., editor, Levels of Selection in Evolution, pages 238-252. Princeton University Press, Princeton, NJ.

Ofria, C., C. Adami, and T. C. Collier. 2003. Selective pressures on genomes in molecular evolution. Journal of Theoretical Biology, 222:477-483. 
Peck, J. 1984. Metapopulation model. Talk given in the Dept. of Biological Sciences, Stanford University.

Peck, J. R. 1992. Group selection, individual selection, and the evolution of genetic drift. The Journal of Theoretical Biology, 159:163-187.

Ray, T. S. 1995. A proposal to create a network-wide biodiversity reserve for digital organisms. http://www.hip.atr.co.jp/ ray/pubs/reserves/node1.html.

Rosenzweig, M. L. 1972. Evolution of the predator isocline. Evolution, 27:84-94.

Roughgarden, J., D. Heckel, and E. R. Fuentes. 1983. Coevolutionary theory and the biogeography and community structure of anolis. In Huey, R., E. Pianka, and T. Schoener, editors, Lizard Ecology: Studies of a Model Organism, pages 371-410. Harvard University Press, Cambridge, Massachusetts.

Schmalhausen, I. I. 1949. Factors of Evolution: The Theory of Stabilizing Selection. University of Chicago Press, Chicago. 1986 edition.

Stoler, D. L., N. Chen, M. Basik, M. S. Kahlenberg, M. A. Rodriguez-Bigas, N. J. Petrelli, and G. R. Anderson. 1999. The onset and extent of genomic instability in sporadic colorectal tumor progression. Proceedings of the National Academy of Science U.S.A., 96(26):15121-15126.

Tomita, M., N. Shimizu, and D. L. Brutlag. 1996. Introns and reading frames: Correlations between splice sites and their codon positions. Molecular Biology and Evolution, 13(9):1219-1223.

Toussaint, M. 2003. Demonstrating the evolution of complex genetic representations: An evolution of artificial plants. In Cantu-Paz, E., J. A. F. Ill, K. Deb, D. Lawrence, and R. Roy, editors, Genetic and Evolutionary Computation Conference (GECCO 2003), pages 86-97. Springer Verlag, 2003.

Trivers, R. 1971. The evolution of reciprocal altruism. Quarterly Review of BIology, 46:35-57.

van Baalen, M. and D. A. Rand. 1998. The unit of selection in viscous populations and the evolution of altruism. Journal of Theoretical Biology, 193:631-648.

van Nimwegen, E., J. P. Crutchfield, and M. Huynen. 1999. Neutral evolution of mutational robustness. Proceedings of the National Academy of Sciences U.S.A., 96: 9716-9720.

Waddington, C. H. 1942. Canalization of development and the inheritance of acquired characters. Nature, 150:563-565.

Wagner, G. P. and L. Altenberg. 1996. Complex adaptations and the evolution of evolvability. Evolution, 50(3):967-976. 
Wagner, G. P., C.-H. Chiu, and T. F. Hansen. 1999. Is hsp90 a regulator of evolvability? Journal of Experimental Zoology (Molecular and Developmental Evolution), 285:116118.

Wallace, D. 1999. Mitochondrial diseases in man and mouse. Science, 283:1482-1488.

Wilson, D. S. 1977. Structured demes and the evolution of group advantageous traits. American Naturalist, 111:157-185.

Wilson, D. S. 1990. Weak altruism, strong group selection. Oikos, 59:135-140.

Wilson, D. S. 1997. Altruism and organism: disentangling the themes of multilevel selection theory. American Naturalist, 150(Supplement):S22-S134.

Wynne-Edwards, V. C. 1962. Animal dispersion in relation to animal behavior. Oliver and Boyd, Edinburgh.

Wynne-Edwards, V. C. 1964. A reply to maynard smith. Nature, 201:1147.

\section{Appendix}

\section{Group Selection Model for limitation of Group-disadvantageous mu-}

\section{tation}

Research Notebook, August 4, 1984

There is a gene which evolves via individual selection but decreases group fitness. There is another gene controlling the rate of introduction via mutation of such "selfish" genes. Given that the selfish gene is introduced, we don't want it to spread over the whole population.

Try Joel Peck's model Peck $(1984,1992)$ of group descent: an infinite number of sites. Groups leave offspring groups with probability $=f$ (\#of selfish). Each generation, selection acts to increase the $\%$ of selfish. The second gene controls the mutation rate to the selfish type.

This model applies not only to behavior but to any trait which may have a population-level negative consequence, or try, a long-term negative consequence.

For the evolution of sex, I imagine that phenotypes causing increased difficulty of producing parthenogens will fix in a sexual population.

Possible difficulty: rates of evolution or selection go to zero as mutation rate goes to 0 . So that always some residual chance of the negative mutation remains. 
Also, one could have a model where a modifier causes the selfish allele to have a selective disadvantage, or from pleiotropy. (Altenberg, 1984a) 